\title{
Special section with selected papers from PODC 2010
}

\author{
Rachid Guerraoui
}

Published online: 22 April 2012

(C) Springer-Verlag 2012

The 29th annual ACM symposium on principles of distributed computing (PODC 2010) was held in Zurich, Switzerland on 25-28 July 2010. The two papers in this special section were chosen by the program committee from the 41 fulllength papers presented at the symposium, based on their quality. In addition to being reviewed, in preliminary form, by the program committee of PODC 2010, the papers were subsequently refereed according to the standard practices of distributed computing. 